\title{
When the Social Becomes Non-Human: Young People's Perception of Social Support in Chatbots
}

\author{
Petter Bae Brandtzaeg \\ University of Oslo, Department of Media and \\ Communication \& SINTEF \\ petterbb@uio.no \\ Kim Kristoffer Dysthe \\ University of Oslo, Institute of Health and Society, \\ Department of General Practice \\ k.k.dysthe@medisin.uio.no
}

\begin{abstract}
Although social support is important for health and well-being, many young people are hesitant to reach out for support. The emerging uptake of chatbots for social and emotional purposes entails opportunities and concerns regarding non-human agents as sources of social support. To explore this, we invited 16 participants (16-21 years) to use and reflect on chatbots as sources of social support. Our participants first interacted with a chatbot for mental health (Woebot) for two weeks. Next, they participated in individual in-depth interviews. As part of the interview session, they were presented with a chatbot prototype providing information to young people. Two months later, the participants reported on their continued use of Woebot. Our findings provide in-depth knowledge about how young people may experience various types of social support-appraisal, informational, emotional, and instrumental support-from chatbots. We summarize implications for theory, practice, and future research.
\end{abstract}

\section{CCS CONCEPTS}

- Human-centered computing; • Human-computer interaction (HCI); • Empirical studies in HCI;

\section{KEYWORDS}

Chatbots, Young people, Artificial Intelligence, Social support

\section{ACM Reference Format:}

Petter Bae Brandtzaeg, Marita Skjuve, Kim Kristoffer Dysthe, and Asbjørn Følstad. 2021. When the Social Becomes Non-Human: Young People's Perception of Social Support in Chatbots. In CHI Conference on Human Factors in Computing Systems (CHI '21), May 08-13, 2021, Yokohama, Japan. ACM, New York, NY, USA, 13 pages. https://doi.org/10.1145/3411764.3445318

Permission to make digital or hard copies of part or all of this work for personal or classroom use is granted without fee provided that copies are not made or distributed for profit or commercial advantage and that copies bear this notice and the full citation on the first page. Copyrights for third-party components of this work must be honored.

For all other uses, contact the owner/author(s).

CHI '21, May 08-13, 2021, Yokohama, Japan

(C) 2021 Copyright held by the owner/author(s).

ACM ISBN 978-1-4503-8096-6/21/05.

https://doi.org/10.1145/3411764.3445318

\author{
Marita Skjuve \\ SINTEF \\ marita.skjuve@sintef.no
}

\author{
Asbjørn Følstad \\ SINTEF \\ asbjorn.folstad@sintef.no
}

\section{INTRODUCTION}

Social support is important for the health and well-being of young people in their emerging adulthood [1] and can be defined as "support accessible to an individual through social ties to other individuals, groups, and the larger community" [2]. National and international studies have indicated that young people increasingly suffer from mental health issues, such as social isolation, anxiety, eating disorders, sexual problems, and depression [3-6]. Despite this, many young people tend not to seek professional help for these problems [7]. Recently, the COVID-19 epidemic has resulted in increased mental health distress among young people and new needs for access to social support online [8,9]. A recent study suggests that young people struggle to access online social support, reporting challenges in navigating and making sense of online information sources [10].

So far, the research on social support has largely focused on how support is given and received in human-human relationships, both offline and online. However, recent advances in technology and artificial intelligence (AI) have facilitated a fundamental shift, where artificial agents, such as chatbots, can provide social support in natural language user interfaces $[8,11]$. This shift is seen in a number of domains from education to therapy, where chatbots are introduced to supplement or replace human agents [13], due to improvements in reasoning and conversational capabilities in chatbots [14].

Chatbots are machine agents with which users interact through natural language dialogue (text, speech, or both) [12]. In this paper, we focus on text-based chatbots for social and mental health purposes, such as Replika, Woebot, and Mitusuku, where a common and important enterprise is not only to provide information to users, but also to enable social relationships to form between the user and chatbot. To do this, the chatbots imitate conversations with friends, partners, therapists, or family members. Such social chatbots are commonly labeled "emotional chatting machines" [13] or "emotionally aware chatbots" [14], and are designed to be humanlike, with the potential to perceive, integrate, understand, and express emotions [13]. Emotional awareness may increase the level of engagement between the user and chatbot. Hence, social chatbots holds immense potential for being a new interface and approach to social support and psychological well-being [15]. 
Although the importance of chatbots as a source of social support is not fully explored in the current research [16], there is a larger body of research in this area that has addressed how artificial agents, such as chatbots, can help older adults in stressful life situations or with more acute health-related concerns. However, there is only a limited amount of research on how chatbots can be a source of everyday social support [17], particularly for young people [18].

When exploring chatbots as a source of social support, it is important to recognize that human-machine relationships can have numerous social and psychological implications [19]. An important issue here is that recent developments in chatbots are due to a massive technology push. This push, with little attention to human needs and experiences, can lead to unintended negative consequences, such as online addiction and loneliness [20]. Chatbots may also present risks, such as biases, inadequate and failed responses [21], and privacy issues [6,22], all of which can negatively affect the quality of the experience of chatbots as a source of social support.

Crucially, there is a lack of in-depth knowledge regarding the types of social support that matter in everyday contexts, the potential risks of non-human support, and how users experience such support from chatbots. This gap in understanding motivates us to investigate how young people perceive chatbots as a source of various forms of social support. Therefore, we pose the following explorative research question:

RQ: How do young people perceive various types (appraisal, emotional, informational, and instrumental) of social support in chatbots, and what are the social implications associated with such chatbot use?

In addressing this question, we offer important exploratory results on how young users experience social support from chatbots and the impact of trust and privacy experiences related to such chatbot use. As the choice of sample in the current study indicates, our focus was not on young people who were struggling with social support related to clinically defined symptoms of mental issues. Rather, the sample was chosen to investigate everyday social support from chatbots that can benefit people in general, not just those struggling with mental health disorders. More specifically, we used in-depth interviews with a sample of young people in their emergent adulthood [18] between 16 and 21 years of age. We also conducted follow-up email interviews of the same participants after a two-month period.

With this qualitative approach, we contribute to the existing literature by investigating the role chatbot use may play in the various forms of perceived social support and psychological experience and well-being in an everyday context among young people. Given the unprecedented ability of chatbots to enhance and access social support at any time and in any place, hence affording greater opportunity for perceived social support, it is both important and relevant to inquire how social support might be experienced as a benefit for young people in an everyday context. Whether chatbots are increasing or decreasing social support could have enormous consequences for society and for young people's well-being. In addition, new knowledge and research on human-chatbot relationships is highly important to guide society, as well as chatbot developers, in a future in which there will be more immediate access to chatbots and $\mathrm{AI}[22]$.

\section{RELATED WORK}

In this section, we start with a brief overview of the theory on social support and then cover related works on young adults before moving on to how social support is found online and through chatbots. We also cover important research that relates to privacy and social support.

\subsection{Theory on Social Support}

Social support is the perception that one has access to care and help through relevant social others [26] such as family, friends, fellow students or colleagues, or therapists. Social support is found to be protective for mental health by improving coping strategies and resistance resources [23-26]. House [27], therefore, suggests studying the quantity and type of social relationships, networks, and the type of support in relation to each other to identify the level of social support gained from different networks [28]. In a human-human context, social support is typically found to consist of four distinct types [27]:

- Appraisal support: The communication of information that is relevant and useful for self-evaluation, offered in the form of feedback, social comparisons, and affirmation.

- Emotional support: The provision of and expression of empathy, love, trust, and caring.

- Informational support: Advice, suggestions, and information provided.

- Instrumental support: The provision of tangible aid and services or goods.

Research from a variety of areas provides evidence of the benefits of forming and maintaining high-quality social connections [29], but the benefits of social connections with machines such as chatbots are less clear. In the current study, we aim to utilize the abovementioned four categories of social support to explore the benefits and limitations of support perceived in social chatbots. In the literature, a distinction has been made between routine and crisis (non-routine) support $[17,25]$. We will focus on the various types of social support in relation to everyday routine rather than on crisis situations.

\subsection{Emerging Adulthood and Social Support}

Social support may be particularly important for young people in their late teens through their twenties. Young people between 18 and 25 years of age are entering a period in life referred to as "emerging adulthood" [30]. This is a distinct period demographically, subjectively, and in terms of identity explorations, parental detachment, and moving away from home [16]. Young people in emerging adulthood are dealing with a range of stress factors-socially, professionally, and/or academically [18]. As such, members of this age group are at a vulnerable time in their development. Reports indicate an increase in mental health issues on a global level among young people [31-33]. 75 percent of major mental disorders have their onset prior to the age of 25 [34]. For these young people, the consequences of insufficient information, preventive efforts, and early interventions can be detrimental [35]. Hence, young people 
have a high need for social support, but they often struggle to reach out for such support.

\subsection{Online and Offline Social Support}

The literature suggests that social support can come from various sources: informal (e.g., family, friends, partners) or more formal (e.g., mental health specialists or community organizations) [36]. The literature also proposes that social support can be available online through apps and sites, such as Instagram [11, 37], Facebook [38], online groups [39], and health forums [40].

Overall, support is an important reason why young people engage online [41], but young people also report challenges in navigating and making sense of those sources [10]. Hence, online support can have disadvantages, such as incorrect information or the exclusion of people with low literacy levels. The Internet and social media are increasingly polluted with misinformation, which can lead to the distribution of harm [42]. It has been argued that the smart use of online chatbots can mitigate the negative effects of health misinformation online and serve as a tool for young people searching for help online [6, 43].

A recent review demonstrated that, under the right conditions, online services can provide useful instrumental and informational social support [46]. Studies have shown that young people report going online frequently to seek out health information [44]. Others have shown that young people with lower well-being are more likely to seek out online support [45]. However, when social support is not received on social media, it could be damaging. For example, in one study, social support sought on Facebook, but not perceived increased adolescents' depressed mood [46].

Differences between online and offline social support have been pointed out in previous research (e.g., [47, 48]). Conflicting evidence exists on whether the time spent on the Internet leads to increased social isolation or strengthened social capital and support [49-51]. Some research, however, posits that online social support is, to an extent, independent of in-person social support [52]. For instance, online support and support communities provide accessibility in terms of time and space, encouraging connections through the weak ties, which may result in better access to diverse information and experts [53]. They also offer an anonymous and private space to participate in self-disclosure [54]. This may also be the case with chatbots.

\subsection{Chatbots and Offline Social Support}

With social chatbots [14], the scope of social support resources has broadened dramatically. Such chatbots may help people who are experiencing troubles and can be interacted with anywhere and anytime [6] while providing comforting and caring effects [55]. They can support people who feel isolated, both emotionally and physically, and provide instant feedback and supportive dialogue [6] Social chatbots designed to deliver cognitive-behavioral therapeutic (CBT) have demonstrated efficacy in treating depression and anxiety in young people [56]. Similarly, receiving empathic responses from a chatbot when talking about emotional subjects have been found to have a positive effect on the user's mood [57].

Although these studies demonstrate that chatbots can potentially be a source of social support, they do not provide detailed insight on how the user qualitatively experiences social support from a chatbot targeting young people. The social implications of chatbots are widely speculated about, and empirical evidence remains rare [58]. A few exceptions are the studies conducted by Kim [61] and Ta et al. [13].

Kim [59] explores teenagers' expectations when interacting with a chatbot intended to support their emotional needs. The participants reported that they expected the chatbot to be a good listener, to serve as a confidential environment where secrets and more personal informational could be shared, and to provide quality advice if needed. Interestingly, the participants reported that they did not want the chatbot to provide soothing or comforting feedback when they shared their challenges but instead to provide encouragement and advice on how to cope.

In a recent study, Ta et al. [17] apply data from user reviews and a questionnaire study to investigate users' experiences of social support when interacting with the social chatbot Replika. Their results reveal that the users perceive the companionship of Replika as a source of everyday social support, particularly emotional, informational, and appraisal support, but not as a source of tangible support.

While these studies offer initial insights on social support in chatbots, they are also limited. Kim's [59] study is based on users' expectations, not experience. Ta et al.'s study [13] is based on user reviews and questionnaire data and are lacking the opportunity for detailed questioning and follow-up that is possible in, for example, interview studies. It may also be noted that, as user reviews are mostly positively worded [60], there may be underreporting of negative aspects. Online reviews are therefore often based on a biased subset of individuals who actually used the service or app, resulting in a two-mode distribution of ratings (non-normal distribution). As such, online reviews may represent data not fully representing the variation of real-life use [61]. Hence, more in-depth knowledge is needed on how young people experience social support from chatbots.

\subsection{Privacy and Trust in Chatbots}

Chatbots for social support may present risks, such as privacy issues $[6,22]$. While storing and processing conversations are necessary for chatbots to provide meaningful replies to messages and share insights and patterns, the need for chatbots to demonstrate confidentiality and integrity has been identified as a barrier to seeking support [62]. Indeed, a safe environment for self-disclosure is crucial [63], an important aspect of social support.

Chatbots are regarded as having substantial potential to serve as an effective tool to support people's self-disclosure [64]. A recent study found that chatbots which provided self-disclosure had a reciprocal effect, promoting deeper participant self-disclosure [64]. Interestingly, self-disclosure plays a central role in the development and maintenance of relationships [65] and social support [66] between humans and may also be true in the context of humanchatbot relationships.

However, self-disclosure and emotional engagement in conversations with chatbots may encourage users to reveal private information [15], including information on health and sexual orientation 


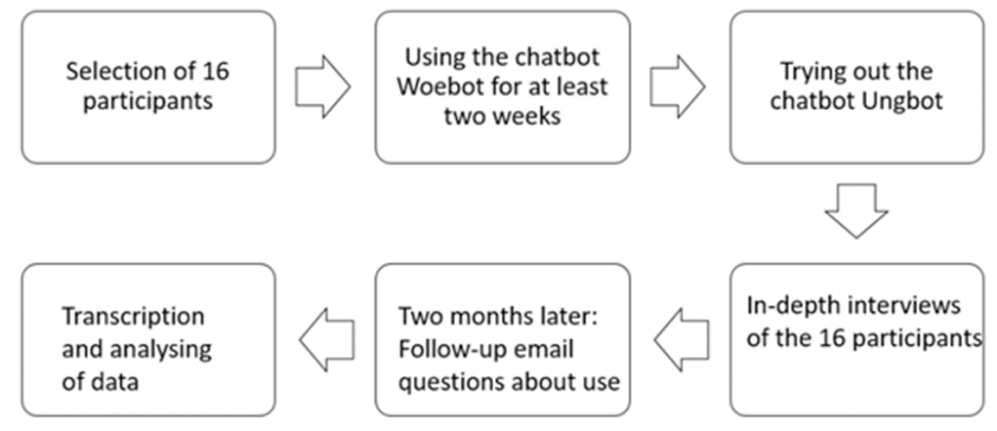

Figure 1: An overview of the different stages in the study

[67]. Previous research on social media has voiced concerns regarding the use of such platforms for information-seeking purposes due to privacy issues [68]. Research on chatbots also indicates a greater level of self-disclosure compared to communicating with humans because chatbots do not experience emotions or make judgments [57].

A lack of trust among users in how a service is storing and sharing personal data can lead the users to stop using the service [69]. Hence, privacy is an important part of the user experience with chatbots [7]. However, little is known about how chatbot users that are seeking social support experience privacy.

\section{METHOD}

In general, the paucity of research examining how chatbot use links to everyday social support is somewhat surprising given the extensive body of research linking social support to health outcomes. To respond to the research question (see page 2), we selected a qualitative approach to achieve an in-depth understanding of how young people experience chatbots as a source of social support. We recruited 16 young participants, who first interacted with a chatbot for mental health (Woebot) for two weeks before participating in individual in-depth interviews. As part of the interview session, they explored a prototype chatbot providing information to young people. Two months later, they reported on their continued use of Woebot. An overview of the study is presented in Figure 1

\subsection{Sample}

Our goal was to investigate everyday social support among young people in their emerging adulthood [18] and to understand why and how this age group experiences various forms of social support from chatbots as well as their reflections on privacy issues. Hence, we decided to target relatively young people between the ages of 16 and 21. Moreover, this age group has vast experience with social media platforms $[51,70]$ hosting chatbots, such as Messenger, Kik, and Telegram. The participants were recruited by giving out handouts with information about the study at various locations in two different Norwegian universities. Norway is regarded as a country with high penetration of digital technologies [71] and was thus a suitable location for this study.
The final sample consisted of 16 young people, which is likely to be regarded as a sufficient number of participants in similar interview studies [72], particularly as we reached data saturation [73]. Nine identified as females and seven as males. All the participants had extensive experience with social media, such as Snapchat, Facebook, Telegram, and Instagram. About half of the sample had experience with chatbots for customer service, and one participant had experience with social chatbots. See Table 1 for an overview of the demographics.

\subsection{Choice of Chatbots: Woebot and Ungbot}

The study participants were presented with two chatbots: Woebot and Ungbot. Woebot is a well-known chatbot that was appropriate for several reasons: it is easy to access, user friendly, and has a strict privacy policy. Woebot conducts CBT-an evidence-based approach for the treatment of various psychological problems-and is designed so that the principles of CBT are utilized in a friendly and engaging manner [56]. The chatbot resembles a friend who checks up on the user's mood and gives advice concerning experiences and worries. Woebot also offers quizzes and videos which are there to help users discover habitual or automatized thought patterns that affect their well-being and mental health. Woebot is mainly a chatbot for mental health support, but it also includes substantial social features, making it a highly relevant chatbot for the purposes of this study. The chatbot not only facilitates socialization with the users but also employs a certain level of human emotional intelligence (i.e., the capability to perceive, integrate, understand, and regulate emotions) [13]. Screenshot examples of Woebot are provided in Figure 2

The young people were presented with the second chatbot, Ungbot, as part of the interview session. This chatbot is an early prototype intended to provide informational support about issues youths may struggle with, including sex, school, bullying, feelings, love, school, and divorce. Available information in the chatbot is drawn from a website for youths (ung.no). As with Woebot, this prototype is also intended to build a relationship with the user over time, although support for this is not implemented. The reason for using Ungbot was to present chatbots with various features to give the participants more insights into how different chatbots work. 
Table 1: Participants (P) age, gender (F/M), and previous experience with chatbots

\begin{tabular}{llll}
\hline Participant \# & Gender & Age & Experience with chatbots \\
\hline P1 & F & 16 & No \\
P2 & F & 18 & No \\
P3 & F & 21 & No \\
P4 & F & 20 & No \\
P5 & M & 19 & Yes, customer service \\
P6 & M & 19 & Yes, customer service \\
P7 & M & 19 & Yes, customer service \\
P8 & M & Yes, customer service \\
P9 & F & 19 & No \\
P10 & F & 19 & No \\
P11 & F & 18 & Yes, customer service \\
P12 & M & 19 & No \\
P13 & M & 19 & Yes, customer service \\
P14 & M & 20 & Yes, customer service \\
P15 & F & 19 & Yes, a social chatbot \\
P16 & F & 20 & Yes, customer service \\
\hline
\end{tabular}

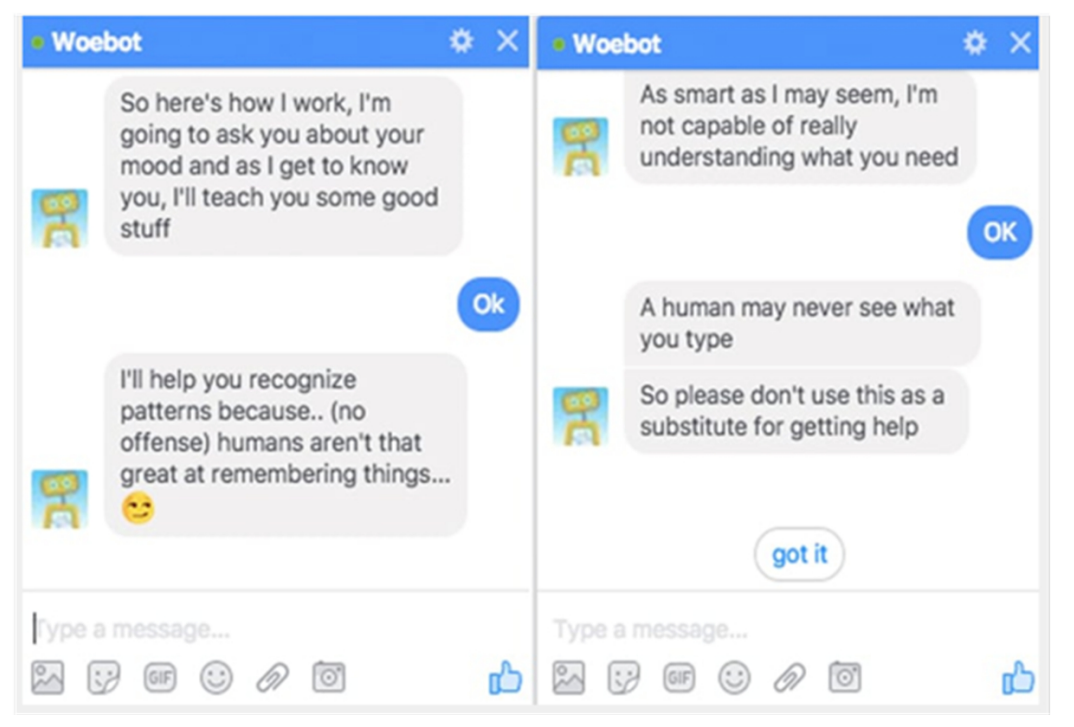

Figure 2: Screenshot of Woebot dialog-explaining to the user how it works as a non-human

\subsection{Procedure}

The participants were invited to use Woebot every day for 14 days and could withdraw from the study at any time. The purpose was not to evaluate the use of Woebot but rather to have the participants use, experience, and get a sense of how chatbots can provide social support. We did not dictate the interaction time or the content of their interactions, seeking to motivate the natural use of the chatbot. After 14 days of use, they participated in an individual interview, conducted face-to-face. As part of the interview session, the participants also tried out a chatbot prototype, Ungbot, targeting young people. Finally, two months later, email interviews were conducted with the participants.
All face-to-face interviews were conducted in a neutral setting, such as a café, on campus, or at a similar informal location chosen by the participants, to make them feel comfortable and safe. The interviews were audio-recorded and transcribed. Most interviews lasted approximately one hour or more (mean: 1 hour, 12 minutes). The participants received NOK 500, approximately 50 US dollars, as compensation. The interviews consisted of three parts. The first part of the interviews focused on open questions concerning the interviewees' general experiences with appraisal, emotional, informational, and instrumental support. Example questions included the following: "When you have problems-who do you go to?" "How do you access information about things that are important to you 
Table 2: Coding scheme used for the analysis of the data

\begin{tabular}{ll}
\hline Code & Description \\
\hline Appraisal support & Appraisal support can be offered in the form of feedback, social comparison, and affirmation. \\
Emotional support & Expressions of empathy, love, trust, and caring. \\
Informational support & Advice, suggestions, and information given by the chatbot to help the user solve a problem. \\
Instrumental support & $\begin{array}{l}\text { Tangible aid, which is characterized by the provision of resources in offering help or assistance in a } \\
\text { tangible and/or physical way, such as providing money or people. }\end{array}$ \\
Privacy and trust & $\begin{array}{l}\text { Experienced privacy issues, such as a feeling of surveillance and data collection, as well as reflections on } \\
\text { sharing personal data with the chatbot. }\end{array}$ \\
\hline
\end{tabular}

concerning difficulties at school or at home?" "Do you find it easy to obtain relevant information or support?"

The second part consisted of an in-depth question about the participant's experiences and reflections concerning chatbots in general, and Woebot in particular, as a source of social support. As a part of the interview session, they also explored the prototype Ungbot to broaden the participants' experience of how social chatbots may work. We questioned their views on the chatbots' ability to provide appraisal, emotional, informational, and instrumental support. We also asked about trust and privacy concerns. In addition, we asked questions about when and where the participants used Woebot as a way to gather more contextual cues to better understand the usage of such chatbots.

Third, after two months, we followed up with an email study. Twelve out of the 16 participants responded. We asked if they had used Woebot or similar chatbots after the interview. If they answered "no," we asked them to explain why, and if the answered "yes," we asked them to share their experiences and motivation for using chatbots.

\subsection{Approvals and Ethical Considerations}

The Norwegian Social Science Data Services approved the study's protocol. All the participants signed a written consent form prior to the interviews. We maintained confidentiality by removing names and identifiable information from the transcripts prior to any analysis. No identifiable information is provided in the excerpts presented in the current paper.

\subsection{Analysis}

We performed a deductive content analysis to identify and investigate patterns within the data [74]. During the first phase of the analysis, we familiarized ourselves with our data by reading through all the transcribed material. We then coded all the data using NVivo 12 pro. The interviews were coded following House et al.'s [27] theoretical framework for social support, with the addition of experienced privacy and trust issues. The coding scheme is presented in Table 2

A meaningful unit could be placed within more than one of the coded categories. For reliability, two researchers were involved in the coding. First, one of the researchers coded all the posts, and then the second went through all the assigned codes. In the event of a disagreement on the coding for a given quote, an alternative code was suggested and subsequently reviewed by the first coder. An alternative coding was suggested for $17 \%$ of the codes in the data. We used a professional translator when translating the quotes from Norwegian to English.

\section{RESULTS}

We have divided our findings mainly under the five main themes: (1) appraisal support, (2) emotional support, (3) information support, (4) instrumental support, and implications, specifically (5) privacy and trust.

\subsection{Appraisal Support}

Most of the participants reported that the chatbots provided some form of appraisal support related to self-evaluation and feedback. Several of the participants explained that the interaction with Woebot, in particular, resulted in greater knowledge about oneself:

"You become in a way, or you may become more familiar with yourself, but that is, it is a little bit difficult to explain..." (P2)

Others noted that the chatbot facilitated self-reflection, further explaining how self-evaluation and knowledge seemed to arise through the process of evaluating the conversation one has with the chatbot:

"Talking to the chatbot is like speaking in a way to yourself, you know that the chatbot is not you, but you feel like it." (P4)

While this perception of having a conversation with oneself through the chatbot could potentially influence the level of honesty and self-disclosure, it could also enable a kind of self-help. As the participant below explains, it feels safe to be honest because you are essentially talking with yourself:

"You can sit at home and be completely alone; it may be easier to be completely honest with something that in a way is not real. Because then you talk to yourself in a way and you are more honest with yourself, than if you sit in a way with someone you do not know so well." (P9)

Some participants explained how Woebot helped them to recognize negative thought patterns. This seemed to occur as a result of Woebot's focus on CBT: asking questions about a topic, which led the participants to reflect further upon how it extends to other aspects of their life, eventually identifying it as negative thought patterns and global labeling:

"It (Woebot) tries to make you aware of your own thoughts. Like, I was chatting (with Woebot) when 
I walked over here. And then it asks, do you ever label yourself, as bad and stuff. Then you say, yes I do. Therefore, you think, if you do have negative thoughts also with others, then you may become a little like that (...), you do it maybe a little too much then. Then you become aware of it. Therefore, it (the chatbot) really just works to clear your mind in a way." (P7)

Some also reported that the chatbot helped them become aware of their emotional state:

"I think what a little cool about it (Woebot) is that when I sometimes talked to him about a little bit of that kind of problem then he asked what emotional state I was in. Also, I have often not thought about that when I face a problem; that I'm I stressed, and nervous." (P9)

This awareness of one's emotions could also be trigged by the chatbot tracking the user's expressions and emotions. This feature provided the participants with an overview of emotional states that were not always known to them:

"It (Woebot) seems like it kind of tracks down your expressions and feelings. It could be that you get someone that know yourself in away. That you get more of an overview then maybe, of what kind of mood and/or self-esteem you have, which in a way I was not aware of before, or which you may not have thought of." (P8)

It was further mentioned that interacting with Woebot is somewhat similar to writing in a diary, a kind of a "me-communication" style, which could further increase reflection about oneself:

"It's like writing on your diary. You get a feeling that you interact and talk to yourself, so you may just think about what you do, like what I have actually done today, what has been nice today in away. And through that you get like that, do not know if you know yourself better then, but you become aware of your own situation." (P15)

\subsection{Emotional Support}

Nearly half the participants reported perceptions of emotional support from Woebot. Some participants felt that the chatbot showed empathy and comfort by, for instance, asking if they were feeling better after a conversation:

"Yes. It (Woebot) eventually asks, after you have talked a bit if you feel better after that conversation. And then you can say either yes or no. If you say no then it continues with other things, or with other techniques that you can try out or so it says that, so good, so nice that I could help you." (P3)

One participant also highlighted that it was safer to approach a chatbot for social and emotional support compared with a human because you could be disappointed in an interaction with a human:

"To a certain extent, you can receive comfort and empathy from a chatbot, but not in the same way as human contact will. Again, I can really speak for myself, but when you are very desperate to get support, and then you do not get the support you really expected or wanted, then it is even more painful than that you had not received support at all. It can be in a way if you just need someone to hear you, even if you know it's a robot talking to you. Therefore, if you are so afraid of somehow getting the rejection at the level of support you want, then it is a safer way to go to a robot." (P16).

Three of the participants said that they forgot that they were talking to a machine, which they reported as positive, potentially strengthening a sense of emotional support:

"I noticed that I might have forgotten a bit that it was a robot. Then you got the feeling that there was actually a person there, to a certain extent, at least. Yes, so then, that was positive." (P8)

Some of the other participants did not experience emotional support from the chatbots. Rather they reported that the chatbot displayed empathy to better understand the user by getting to know their perspective, as noted by the participant below:

"I feel it is more that the chatbot can, that it can be, the person you can talk to, and that you feel that you have a conversation and it can give you different tips and such. However, I feel it will be difficult for it to actually manage to comfort you." (P6)

\subsection{Informational Support}

Most of the participants regarded chatbots as easy, available, and even trusted sources of informational support. This was seen for both Woebot and the Ungbot prototype:

"I think if you are struggling with something mental and need advice and tips, then I think maybe a chatbot can help you quite a bit (...). Give you advice on, for example, a page on ung.no (youth information website) where there is a lot of information, who you can contact, the health nurse, whether it is a health station or something, not true." (P11)

A key feature of chatbots is that they are unconstrained by time and space. The participants seemed to value this aspect and noted that they, for example, could sit on the subway or in their own room receiving informational support:

"I sat in my room alone talking to the chatbot and you stay in a way, you get such peace of mind (...) if you go to a psychologist, for example, it is often a kind of office-like place." (P1)

Several of the participants also appreciated that the information was provided in an immediate and efficient fashion:

"Although it is not completely interactive as a human being, as such a proper conversation, so it guides me in a way on the right track and can provide relevant information as, well, yes by which button you press in relation to how you have it that day. And then it asks several questions where it's a bit like that, you 
press yes, no, or I understand this, tell me more about this. Then it comes with information." (P3)

Another also regarded both Ungbot and Woebot as chatbots that not only guided the user but also actively approached the user with information in a proactive manner:

"They (Ungbot/Woebot) were kind of searching or approaching you in a way, and I think it's a little reassuring for someone who might have trouble talking to someone or feel like they do not want to talk or ask questions. Then it's kind of good that those chatbots is very like that, answers right away, asks questions all the time because then you sort of continue, instead of having to wait for answers like that and that you actually talk to a person." (P6)

The participants expressed trust in the information provided by the two chatbots. They reported that the information was of high quality. Further, they explained that their friends sometimes lacked the ability to provide such factual knowledge:

"There are things Woebot knows that my friends do not know. I think if I had talked about anxiety with a friend, I might have had stories about personal experiences. And what they somehow felt themselves and how they coped with it. But Woebot was not, Woebot had a more concrete and factual information." (P10)

Moreover, many young people report a barrier to visiting a specialist, a parent, or a friend because it can be embarrassing. Hence, it is safer to ask a chatbot, as one of the participants explained:

"Young people are quite scared, you could say, in order to get to the health nurse, to seek help then, so I think maybe a robot would have been the best. A robot is behind a screen." (P1)

\subsection{Instrumental Support}

Although a chatbot is not a physical entity and therefore is not able to provide tangible support per se, some of the participants did report that the chatbots could provide tangible aid, such as helping them contact psychologists, show them where to look for information, or urge them to contact friends. This indicates that a chatbot may have the potential to help solve practical problems:

"Yet, actually I believe that such chatbots (Woebot and Ungbot) can help you to get in touch or motivate you to contact help, if needed." (P7)

However, the advice given by the chatbot might not be possible to achieve or be appreciated by the user:

"Imagine if it's a person who does not have that many friends then the chatbot says that you should go and talk to a friend. It can be really hurtful." (P14)

The reason for the lack of willingness to adhere to advice from the chatbot may be related to a lack of trust. For example, one participant explained that she needed to become more familiar with that chatbot to act on the advice it gave her:

"Yes, if you actually create a long-term relationship with it (the chatbot), then you are familiar with it and you know in away how it works. And if you somehow actually lean to know that robot and actually talk to it and get help and recommends you go to a psychologist, so I think I would have done it, yes." (P3)

Although several participants reported social support on various levels, they also reported limitations in this support because chatbots are non-human entities. However, some participants found it helpful that Woebot clearly informed the participants about what to expect in terms of help and support and that it had limitations:

"It's very good that it (Woebot) in a way clarifies its limitations. Therefore, you know it before you start with it. And when you know it and it in a way says"now I cannot help you," then you understand almost logically that then I should pass it on to someone else." (P5)

\subsection{Privacy and Trust}

Most of the young people in this study reported no serious privacy concerns. The participants expressed a feeling of freedom and anonymity in talking with the chatbot.

However, when we followed up on privacy issues in the interviews, some expressed thoughts about potential scenarios where the information shared could be misused:

"I do not care (about privacy) because I feel I have nothing to be concerned about I think (...). However, it can happen that someone goes, like one of those who control the robot I was about to say, goes into the chatbot, they probably have access to conversations." (P9)

Others reported that they trusted chatbots more than humans, though also acknowledging privacy concerning in chatbots:

"P3: Robot is much more to be trusted I feel, but. ...

INT: You trust chatbots more?

P3: Yes, more than humans.

INT: Because?

P3: Because humans may miss and talk to others sometimes, true. But on the other hand, it may be that someone else who does not have access gets access to those systems, to what you have written, for example."

One participant described the chatbot as a kind of "safe space" where you could go to and feel free because the conversation was not archived:

"For me it was there that I experienced at least that there, then, as long as there is not a person there then it will be in a way, which conversation will be there and then. It's like no one remembers it, or, yes, if you understand, then you feel more free." (P8)

Another participant argued that there were no real privacy issues as long as the chatbot was not compromised because there was already so much data around:

"As long as the chatbot is not part of a corrupt scheme. But I think it (privacy) might be okay. I think there is so many data, already so much floating around so it does not matter that much " (P7) 


\subsection{Findings from Follow-up Email Interviews}

After two months, six of the 12 participants reported continued use of Woebot. Three also reported having tried out other social chatbots, such as Mitsuku and Replika. One of the participants, who reported being in a period of reflection and questions regarding sexual orientation, reported continued use of Woebot:

"I have used Woebot and think it is good. It encourages me a lot, and I think many people can enjoy it to handle both negative and positive situations in life." (P3)

Another participant reported that he had used Woebot because he felt depressed and was not able to talk to his parents or friends about his situation. He also expressed that he would have used Woebot even before the current study if he had been aware of it. Woebot supported him in self-reflection and affect labeling when talking about his personal problems:

"Woebot helps me to learn to think differently and

help me to cope with my negative thoughts." (P6)

Overall, the participants who reported continued use of the chatbots seemed to be in particular need of social support to cope with issues or concerns in their everyday life. The participants who did not report continued use noted that they would rather seek social support from friends and others and did not need a chatbot to share with or talk to.

\section{DISCUSSION}

Users' engagement with chatbots for social support is a subject that requires thoughtful reflection from HCI (Human-Computer Interaction) researchers and practitioners. First, the actual user experience and whom and how such chatbots are used are not well understood, which includes the social implications of such use. The current paper presents an in-depth analysis of how a sample of young people experience support from social chatbots, here exemplified by a mental health chatbot Woebot and a prototype chatbot for information provision, as well as potential social implications.

Second, previous research has tended to focus on the use of social chatbots by people in need of mental health support as well as older adults. The everyday context of social support has been somewhat neglected, particularly among young people who are in their emerging adulthood and require social support. Ta et al. [17] reports interesting initial findings on social support. Our study contributes to those findings, drawing on data that provide in-depth information on how and why different types of social support occur in human-chatbot relationships.

However, the results of the present research support many of the conclusions of earlier studies. For instance, the low threshold and easy access to social support in using a chatbot [6] was evident in the present research. Moreover, consistent with prior chatbot research, the perception of social support was frequently reported by the participants (e.g. [17]). The use of in-depth user interviews enabled us to probe the exact nature of perceived social support when using chatbots and the implications of such use.

The results of the present study suggest that social support is perceived because of three main experiences and functions when interacting with social chatbots. First, young people reported that the chatbots could give them easy access to social support on their own terms and in their own preferred context. For example, some reported that the context of chatbot use was sitting at home in their own bed, noting this as a safe space behind the screen for dealing with more difficult things when interacting with the chatbot. In addition, it was easily available $24 / 7$.

Second, communicating with a chatbot was reported to be less of a barrier than interacting with a human (friend, family, therapist, etc.). Typically, the young people in the current study reported that they found it easier to self-disclose to a chatbot than to a human. A main reason for this was that they felt that the chatbot was not judging them for their weaknesses and struggles because it was regarded as non-human support. They reported that chatbots provide both anonymity and privacy, creating a space for conversation and confession that may support self-reflection and affect labeling (putting feelings into words). We know from previous research that young people experience barriers to seeking help. For example, in a social media context, judging and shaming are part of what young people can experience and fear when seeking help or support [75]. Young people tend to be reluctant to seek professional help, even if they are in need of mental health support [7]. Hence, social chatbots may be an efficient and low-threshold alternative allowing young people to talk more freely about their problems in the early stages of these problems.

Third, in line with the principles of CBT young people also experienced that social chatbots could help them assess themselves and think differently about their own problems. An interesting finding from our study is that the chatbot seemed to facilitate appraisal support by encouraging self-reflection. In human-human interactions, appraisal support is thought to include aspects like receiving validation and support for one's feeling and thoughts [32]. In chatbot interaction, the process of appraisal support may be different. In the current study, the participants said that the chatbot encouraged them to talk to themselves, such as writing a diary in an interactive mode. It seemed like the process of providing data to the chatbot made the participants discover and evaluate characteristics and solutions concerning themselves, their emotions, or their thoughts that they were unaware of prior to the interaction. This finding is in line with Ta et al. [17], who also find that the relationship with social chatbots can lead to strengthened introspection. In our study, some of this appraisal support was explained by the experience of the chatbot as knowledgeable, at least on a general level. Some of the participants also reported that they trusted the chatbot more than friends, as a chatbot was better informed regarding informational support and different issues and had new ways of thinking. Therefore, a chatbot may contribute to less noise in navigating and making sense of online information sources (e.g., [10]).

While the social implications of this low-threshold contact with the chatbot for social support are interesting and may lead to increased awareness of the users' own actions and thoughts through appraisal support, it has potentially important implications for how young people relate to others and understand their self-and how they struggle and choose to cope with it. In addition, chatbots may be an excellent way to reach young people that fits their growing individuality, independence, and mastery, encouraging and supporting young people to seek necessary professional help early for their developing mental health difficulties [7]. Chatbots may 
also be a useful and accessible source to social support during the COVID-19 pandemic, lessening the psychological harm caused by fear and isolation. The lockdown and social distancing policy during COVID-19 may have hampered the possibilities to receive real-life social support from friends and professionals. Recent studies also show that the pandemic has resulted in increased mental health distress among young people $[8,9]$.

An important finding, however, is that chatbots as a source of social support do not suit all young people. Some young people in our sample reported that they would prefer friends over a chatbot and that they saw the chatbot as an alternative mainly for people without a social network or who were experiencing troubles. For instance, not all our participants believed that chatbots were empathic or emotional. Cobb [23] states that emotional support involves, among other things, "information leading the subject to believe that he is care for and loved" (p. 300) or "information leading the subject to believe that he is esteemed and valued" (p. 300). The notion of "believing" might be important. That is, it might be difficult for a chatbot to make the user believe that the user is loved and valued by the chatbot.

Although previous research has found that social chatbots can make the user feel loved [17] and that the robot's request for care may be perceived as real [76], our results show that this is not the case for everyone. Similar to Ta et al.'s study [17], we find that some may perceive artificial agents to be a less effective source of social support than others. However, some of our participants reported that such variation could in part be due to the level of familiarity with the chatbot; that is, knowing the chatbot well. It requires time to develop relationships with a chatbot, as it takes time to develop relationships with humans. Prior research seems to overlook the role of social connectedness, that is, the human requirement to establish and develop social relationships, which is a fundamental human requirement [77]. This demonstrates the complexity of both the human-chatbot interaction and the construct of social support, further exemplifying that young people have different needs and experiences when it comes to chatbots. For example, not everyone is in need of an artificial agent as a source of social support, but those who are may require time to develop social relationships with the machine. Chatbots provide young people with a new opportunity to be connected, to increase their chances of satisfying their social needs, and to access social support. Understanding how people develop social relationships (e.g., the social penetration theory [76]) with chatbots can provide new insights into the area of HCI. Future chatbots may provide enhanced social or communicational competence to enable human-chatbot relationship development [78]. Further investigation of those crucial issues is needed.

\subsection{Implications for Design and Practice}

The designers of chatbots intended for social support should consider the varied uses and experiences reported by users and recognize that not all users have the same uses of a chatbot, nor do they derive the same experiences from their use. For instance, there are clear distinctions between the uses of a chatbot to maintain various forms of social support. Informational support and appraisal support require more efficient and knowledgeable content delivery, while emotional support requires more humanlike support. Chatbots should also be designed for relationship development, which may require a broader focus on social competence and communication competence.

When it comes to privacy and trust, the participants in our study did not report the same problems with chatbots that they report with other apps and social media [69], despite the fact that chatbots collect user data and conversations related to social support issues. This finding may be surprising, in particular as interactions with social chatbots may lead users to share highly personal and sensitive information. We are not certain if we can explain this finding well, or of this finding is indeed representative of youth in general. Possibly, the lack of privacy concerns could be due to the research design and the age group. Another explanation could be that young people - like other users - have not yet been sensitized to possible privacy concerns in social chatbots through media reports or exchanges in social media. If this latter explanation is valid, it may be that social chatbot developers are at a point in time where it is of particular importance to demonstrate trustworthiness in information safety and privacy protection, so as to not risk using user trust due to privacy issues in the future.

Another challenge, related to that of trust and privacy, is that the availability of and restrictions on data will shape the interactions with the user as well as the level of social support. Although the participants in the current study did not mention this, it is a potential implication for chatbot users. Despite inherent shortcomings in the data itself, some chatbots can make authoritative and definitive knowledge claims and responses to users, which may affect the social support the users receive. However, these applications currently tend to rely on serious companies working for the social good rather than companies aiming for money and power. Thus, the investment of time and effort in social chatbots may create opportunities that could bring in more companies looking only for profit. Accordingly, there might be a need for more strict privacy regulations for chatbots that develop social relationships with their users.

\subsection{Limitations and Further Research}

The present research investigates how young people experience chatbots as a source of social support. An important limitation is the relatively small and self-selected sample, which constrains the generalizability of the results. How users experience chatbots as a source of social support might change across regions, cultures, and user groups. A second limitation is the retention rate for the email interviews, where 12 of the 16 participants responded, which makes the follow-up part of the study vulnerable to bias.

Future research should study a wider group of participants, for example to identify patterns of usage among a greater sample over time. This would allow for investigating the generality of the presented findings, and also to research the development of chatbot use and experience over longer time periods. In particular, it would be of interest to see how peoples' chatbot use and need for social support develop when using chatbots for prolonged periods of time and how frequency of use is motivated by various forms of social support. There is, for instance, considerable research in the field of 
habit formation that could inform the study of chatbot use, looking more in depth at how habit affects continued use.

HCI research should also consider how users' desire to selfdisclose to chatbots can be accommodated in a privacy-protecting manner. At present, social chatbots may not offer fine-grained options for privacy control as users have grown used to in, for example, social media services (e.g. Facebook) or browsers (e.g. Chrome). Based on the results of previous Facebook research [70], it would seem that users are changing the default privacy settings in a motivated manner, which may also be something that chatbot users would like when their usage matures. The present study only collected privacy experiences from users. It would be valuable also to conduct research that examines chatbot privacy settings, terms of use, and user data flows in a more technical way (e.g., [69]).

\section{CONCLUSION}

The current study contributes to the understanding of how young people in emerging adulthood perceive social chatbots, particularly how various types of social support were experienced over time in an everyday context, as well as trust and privacy issues related to such use. A key finding is that chatbots may be experienced as sources of appraisal, informational, emotional, and instrumental support mainly because of their low-threshold character and because they are perceived to offer a safe and anonymous space for conversation and confession. This is partly due to the context of use; users can get social support from the social chatbot at any place and time, even from their bedroom in the middle of the night. In addition, the interaction with a chatbot, as a non-human actor, provides a refuge from social judgment which in turns stimulates self-disclosure. Moreover, chatbots supporting self-reflection or affect labeling may help users to think more constructively about their own problems. Social chatbots are also perceived as an efficient service to access trusted information support. Finally, chatbots for social support appeared to be mainly useful over time for young people who reported some kinds of problems or struggles in their everyday life. Interestingly, privacy issues regarding social chatbots were not reported by the participants in this study, despite the potential for self-disclosure when using such chatbots. Design considerations for such technologies are suggested to improve user experiences with chatbots for social support.

\section{ACKNOWLEDGMENTS}

This work was supported the Norwegian Research Council, grant agreement no 262848. We would also like to thank Anja Vranic at the University of Oslo for helping out with the coding process.

\section{REFERENCES}

[1] Lene Arnett Jensen. 2011. Bridging Cultural and Developmental Approaches to Psychology: New Syntheses in Theory, Research, and Policy. Oxford University Press, New York, NY.

[2] Nan Lin, Walter M. Ensel, Ronald S. Simeone, and Wen Kuo. 1979. Social support, stressful life events, and illness: A model and an empirical test. F. Health. Soc Behav. 20, 2, (Jun. 1979), 108-119. DOI: https://doi.org/10.2307/2136433

[3] Eve Griffin and Elaine Mcmahon. 2020. Adolescent mental health: Global data informing opportunities for prevention. EClin. Med. 24 (Apr. 2007). DOI: https: //doi.org/10.1016/j.eclinm.2020.100413

[4] Helen Bould, Becky Mars, Paul Moran, Lucy Biddle, and David Gunnell. 2019 Rising suicide rates among adolescents in England and Wales. Lancet 394, 10193 (Jul. 2019), 116-117. DOI: https://doi.org/10.1016/S0140-6736(19)31102-X
[5] Jean M. Twenge. 2020. Increases in depression, self-harm, and suicide among US adolescents after 2012 and links to technology use: Possible mechanisms. Int. J. Psychiatry Clin. Pract. appi. prcp. 20190015 (Mar. 2020), 19-25. DOI: https: //doi.org/10.1176/appi.prcp.20190015

[6] Marita Skjuve and Petter Bae Brandtzæg. 2018. Chatbots as a new user interface for providing health information to young people (pp. 59-66), in Andersson, Y., Dahlquist, U., Ohlsson, J. (Eds.). Youth and News in a Digital Media Environment - Nordic-Baltic Perspectives. Retrived from https://www.nordicom.gu.se/sites/ default/files/kapitel-pdf/06_bjaalandskjuve_brandtzaeg.pdf

[7] Debra J. Rickwood, Frank P. Deane, and Coralie J. Wilson. 2007. When and how do young people seek professional help for mental health problems? Med. 7. Aust. 187, S7 (Oct. 2007), S35-S39. DOI: https://doi.org/10.5694/j.1326-5377.2007.tb01334.x

[8] Lucie Cluver. 2020. Solving the global challenge of adolescent mental ill-health. Lancet Child Adolesc. Health 4, 8 (Aug. 2020), 556-557. DOI: https://doi.org/10. 1016/S2352-4642(20)30205-4

[9] Louise Dalton, Elizabeth Rapa, and Alan Stein. 2020. Protecting the psychological health of children through effective communication about COVID-19. Lancet Child Adolesc. Health 4, 5 (May 2020), 346-347. DOI: https://doi.org/10.1016/S23524642(20)30097-3

[10] Camilla Gudmundsen Høiland, Asbjørn Følstad, and Amela Karahasanovic 2020. $\mathrm{Hi}$, can I help? Exploring how to design a mental health chatbot for youths? Hum. Technol. 16, 2 (Aug. 2020), 139-169. DOI: https://doi.org/10.17011/ht/urn. 202008245640

[11] Chia-Fang Chung, Elena Agapie, Jessica Schroeder, Sonali Mishra, James Fogarty, and Sean A Munson. 2017. When personal tracking becomes social: Examining the use of Instagram for healthy eating. In Proceedings of the 2017 CHI Conference on Human Factors in Computing Systems, ACM Inc., New York, NY, 1674-1687. https://doi.org/10.1145/3025453.3025747

[12] Asbjørn Følstad and Petter Bae Brandtzaeg. 2020. Users' experiences with chatbots: findings from a questionnaire study. Quality and User Experience. 5,3 (Apr. 2020), 14 pages. DOI: https://doi.org/10.1007/s41233-020-00033-2

[13] Hao Zhou, Minlie Huang, Tianyang Zhang, Xiaoyan Zhu and Bing Liu. 2017. Emotional chatting machine: Emotional conversation generation with internal and external memory. arXiv preprint arXiv:1704.01074 (May 2017). Retrieved June 22, 2020, from https://www.arxiv-vanity.com/papers/1704.01074/

[14] Endang Wahyu Pamungkas. 2019. Emotionally-aware chatbots: A survey. arXiv preprint arXiv:1906.09774 (Jul. 2019). Retrieved June 2, 2020, from https://arxiv. org/pdf/1906.09774.pdf

[15] Jaya Narain, Tina Quach, Monique Davey, Hae Won Park, Cynthia Breazeal, and Rosalind Picard. 2020. Promoting wellbeing with Sunny, a chatbot that facilitates positive messages within social groups. In Extended Abstracts of the $2020 \mathrm{CHI}$ Conference on Human Factors in Computing Systems. ACM Inc., New York, NY, 1-8. https://doi.org/10.1145/3334480.3383062

[16] Emily G. Lattie, Rachel Kornfield, Kathryn E. Ringland, Renwen Zhang, Nathan Winquist, and Madhu Reddy. 2020. Designing mental health technologies that support the social ecosystem of college students. In Proceedings of the $2020 \mathrm{CHI}$ Conference on Human Factors in Computing Systems (CHI'2020). ACM Inc., New York, NY, 1-15. https://doi.org/10.1145/3313831.3376362

[17] Vivian Ta, Caroline Griffith, Carolynn Boatfield, Xinyu Wang, Maria Civitello, Haley Bader, Esther Decero, and Alexia Loggarakis. 2020. User experiences of social support from companion chatbots in everyday contexts: Thematic analysis. f. Medical Internet Res. 3 (March 2020), e16235. DOI: https://doi.org/10.2196/16235

[18] Jeffrey Jensen Arnett. 2011. Emerging adulthood(s): The cultural psychology of a new life stage. In Jensen, L. A. (Ed.), Bridging Cultural and Developmental Approaches to Psychology: New Syntheses in Theory, Research, and Policy (p. 255275). Oxford University Press, Oxford

[19] Shyam Sundar. 2020. Rise of machine agency: A framework for studying the psychology of human-AI interaction (HAII). F. Comput.-Mediat. Commun. 25, 1 (Jan. 2020), 74-88. DOI: https://doi.org/10.1093/jcmc/zmz026

[20] Asbjørn Følstad, Petter Bae Brandtzaeg, Tom Feltwell, Effie L. C. Law, Manfred Tscheligi, and Ewa A. Luger. 2018. SIG: Chatbots for social good. In Extended Abstracts of the 2018 CHI Conference on Human Factors in Computing Systems. ACM Inc., New York, NY, 1-4. https://doi.org/10.1145/3170427.3185372

[21] Linett Simonsen, Tina Steinstø, Guri Verne, and Tone Bratteteig. 2020. "I'm disabled and married to a foreign single mother." Public service chatbot's advice on citizens' complex lives. In Hofmann, S. et al. (Eds.) Electronic Participation. ePart 2020 (pp 133-146). Lecture Notes in Computer Science, Vol. 12220. SpringerVerlag, New York, NY. https://doi-org-443.webvpn.fjmu.edu.cn/10.1007/978-3030-58141-1_11

[22] Asbjørn Følstad and Petter Bae Brandtzæg. 2017. Chatbots and the new world of HCI. Interactions 24, 4 (Jul. 2017), 38-42. DOI: https://doi.org/10.1145/3085558

[23] Sidney Cobb. 1976. Social support as a moderator of life stress. Psychosom. Med. 38, 5 (Oct. 1976), 300-314. DOI: https://doi.org/10.1097/00006842-197609000-00003

[24] Sheldon Ed Cohen and S. L. Syme. 1985. Social Support and Health. Academic Press, Orlando

[25] Nan Lin, Xiaolan Ye, and Walter M. Ensel. 1999. Social support and depressed mood: A structural analysis. F. Health Soc. Behav. 4, 4 (Dec. 1999), 344-359. DOI: https://doi.org/10643160 
[26] Ellen Selkie, Victoria Adkins, Ellie Masters, Anita Bajpai, and Daniel Shumer. 2020 Transgender adolescents' uses of social media for social support. f. Adolesc. Health. 66, 3 (Mar. 2020), 275-280. DOI: https://doi.org/10.1016/j.jadohealth.2019.08.011

[27] James S. House, Robert L. Kahn, J.D. Mcleod, and D. Williams. 1985. Measure and concepts of social support. In Cohen, S. \& Syme, S.L. (Eds.), Social Support and Health (pp. 83-108). Academic, New York, NY.

[28] James S. House. 1987. Social support and social structure. Sociological Forum 2, 1 (Dec. 1987), 135-146. DOI: https://doi.org/10.1007/BF01107897

[29] Robert D. Putnam. 2000. Bowling Alone: The Collapse and Revival of American Community. Simon \& Schuster, New York, NY.

[30] Jeffrey Jensen Arnett. 2000. Emerging adulthood: A theory of development from the late teens through the twenties. Am. Psychol. 55, 5 (May 2000), 469-480. DOI: https://doi.org/10.1037/0003-066X.55.5.469

[31] Alize J. Ferrari, Fiona J. Charlson, Rosana E. Norman, Scott B. Patten, Greg Freedman, Christopher J. L. Murray, Theo Vos, and Harvey A. Whiteford. 2013. Burden of depressive disorders by country, sex, age, and year: findings from the global burden of disease study 2010. PLoS Med. 10, 11 (Nov 2013), e1001547. DOI https://doi.org/10.1371/journal.pmed.1001547

[32] Harvey A. Whiteford, Louisa Degenhardt, Jürgen Rehm, Amanda J. Baxter, Alize J. Ferrari, Holly E. Erskine, Fiona J. Charlson, Rosana E. Norman, Abraham D. Flaxman, and Nicole Johns. 2013. Global burden of disease attributable to mental and substance use disorders: Findings from the Global Burden of Disease Study 2010. Lancet 382, 9904 (Nov. 2013) 1575-1586. DOI: https://doi.org/10.1016/S01406736(13)61611-6

[33] Richard J. Hazler and Sharon A. Denham. 2011. Social isolation of youth at risk: Conceptualizations and practical implications. 7. Couns. Dev. 4 (Dec. 2011) 403-409. DOI: https://doi.org/10.1002/j.1556-6678.2002.tb00206.x

[34] Julia Kim-Cohen, Avshalom Caspi, Terrie E. Moffitt, Honalee Harrington, Barry J. Milne, and Richie Poulton. 2003. Prior juvenile diagnoses in adults with mental disorder: Developmental follow-back of a prospective-longitudinal cohort. Arch Gen. Psychiatry. 60, 7 (Jul. 2003), 709-717. DOI: https://doi.org/10.1001/archpsyc. 60.7.709

[35] Randy P. Auerbach, Philippe Mortier, Ronny Bruffaerts, Jordi Alonso, Corina Benjet, Pim Cuijpers, Koen Demyttenaere, David D. Ebert, Jennifer Greif Green, and Penelope Hasking. 2018. WHO World Mental Health Surveys International College Student Project: Prevalence and distribution of mental disorders. F. Abnorm. Psychol. 127, 7 (Oct. 2018), 623-638. DOI: https://doi.org/10.1037/abn0000362

[36] Shelley E. Taylor. 2011. Social support: A review. In Friedman, H. S. (Ed.).The Oxford Handbook of Health Psychology (pp. 189-214). Oxford University Press, New York, NY

[37] Nazanin Andalibi, Pinar Ozturk, and Andrea Forte. 2017. Sensitive self-disclosures, responses, and social support on Instagram: The case of\# depression. In Proceedings of the 2017 ACM Conference on Computer Supported Cooperative Work and Social Computing (CSCW'2017). ACM Inc., New York, NY, 1485-1500. https: //doi.org/10.1145/2998181.2998243

[38] Eline Frison and Steven Eggermont. 2020. Toward an integrated and differential approach to the relationships between loneliness, different types of Facebook use, and adolescents' depressed mood. Commun. Res. 47, 5 (Dec. 2015), 701-728. DOI: https://doi.org/10.1177/0093650215617506

[39] Marsha White and Steve M. Dorman. 2001. Receiving social support online: implications for health education. Health Educ. Res. 16, 6 (Dec. 2001), 693-707. DOI: https://doi.org/10.1093/her/16.6.693

[40] Per E. Kummervold, Deede Gammon, Svein Bergvik, Jan-Are K. Johnsen, Toralf Hasvold, and Jan H. Rosenvinge. 2002. Social support in a wired world: Use of online mental health forums in Norway. Nord. F. Psychiatry 56, 1 (Jul. 2009), 59-65. DOI: https://doi.org/10.1080/08039480252803945

[41] Claudette Pretorius, Derek Chambers, and David Coyle. 2019. Young people's online help-seeking and mental health difficulties: Systematic narrative review. 7 . Medical Internet Res. 21, 11 (Nov. 2019), e13873. DOI: https://doi.org/10.2196/13873

[42] Cristina M Pulido, Laura Ruiz-Eugenio, Gisela Redondo-Sama, and Beatriz Villarejo-Carballido. 2020. A new application of social impact in social media for overcoming fake news in health. Int. 7. Environ. Res. Public Health. 17, 7 (Mar. 2020), 2430. DOI: https://doi.org/10.3390/ijerph17072430

[43] Catrin Sohrabi, Zaid Alsafi, Niamh O'neill, Mehdi Khan, Ahmed Kerwan, Ahmed Al-Jabir, Christos Iosifidis, and Riaz Agha. 2020. World Health Organization declares global emergency: A review of the 2019 novel coronavirus (COVID-19). Int. 7. Surg. 76 (Apr. 2020), 71-76. DOI: https://doi.org/10.1016/j.ijsu.2020.02.034

[44] Sylvia Deidre Kauer, Cheryl Mangan, and Lena Sanci. 2014. Do online mental health services improve help-seeking for young people? A systematic review. F. Medical Internet Res. 16, 3 (Mar. 2014), e66. DOI: https://doi.org/10.2196/jmir.3103

[45] Victoria Rideout and Susannah Fox. 2018. Digital health practices, social me dia use, and mental well-being among teens and young adults in the US. Articles, Abstracts, and Reports 1093, 96 pages. Retrieved May 27, 2020 from https://digitalcommons.psjhealth.org/publications/1093

[46] Eline Frison and Steven Eggermont. 2015. The impact of daily stress on adolescents' depressed mood: The role of social support seeking through Facebook. ? Comput. Hum. Behav. 44, 11 (Mar. 2015), 315-325. DOI: https://doi.org/10.1016/j. chb.2014.11.070

[47] Paula Klemm, Melanie Hurst, Sandra L. Dearholt, and Susan R. Trone. 1999. Gender differences on Internet cancer support groups. Comp. Nurs. 17, 2 (Mar, 1999), 65-72. Retrieved June 27, 2020 from https://pubmed.ncbi.nlm.nih.gov/ 10194883 /

[48] Ulrike Pfeil, Panayiotis Zaphiris, and Stephanie Wilson. 2009. Older adults' perceptions and experiences of online social support. Interact. Comput. 21, 3 (Jul. 2009), 159-172. DOI: https://doi.org/10.1016/j.intcom.2008.12.001

[49] Norman H. Nie. 2001. Sociability, interpersonal relations, and the Internet: Reconciling conflicting findings. Am Behav Sci. 45, 3 (Nov. 2001), 420-435. DOI: https://doi.org/10.1177/00027640121957277

[50] Petter Bae Brandtzæg. 2012. Social networking sites: Their users and social implications-A longitudinal study. f. Comput.-Mediat. Commun.17, 4 (Sept. 2012), 467-488. DOI: https://doi.org/10.1111/j.1083-6101.2012.01580.x

[51] Candice L Odgers and Michaeline R Jensen. 2020. Annual Research Review: Adolescent mental health in the digital age: Facts, fears, and future directions. $\mathcal{f}$ Child Psychol Psychiatry. 61, 3 (Jan. 2020), 336-348. DOI: https://doi.org/10.1111/ jcpp. 13190

[52] David A. Cole, Elizabeth A. Nick, Rachel L. Zelkowitz, Kathryn M. Roeder, and Tawny Spinelli. 2017. Online social support for young people: Does it recapitulate in-person social support; can it help? Comput. Hum. Behav. 68, 3 (Mar. 2017), 456-464. DOI: https://doi.org/10.1016/j.chb.2016.11.058

[53] Barry Wellman and Keith Hampton. 1999. Living networked on and offline. Contemp Sociol. 28, 6 (Nov. 1999), 648-654. DOI: https://doi.org/10.2307/2655535

[54] Xing Zhang, Shan Liu, Xing Chen, Lin Wang, Baojun Gao, and Qing Zhu. 2018. Health information privacy concerns, antecedents, and information disclosure intention in online health communities. Inf. Manag. 55, 4 (Jun. 2018), 482-493. DOI: https://doi.org/10.1016/j.im.2017.11.003

[55] Minha Lee, Sander Ackermans, Nena Van As, Hanwen Chang, Enzo Lucas, and Wijnand Ijsselsteijn. 2019. Caring for Vincent: A chatbot for self-compassion. In Proceedings of the 2019 CHI Conference on Human Factors in Computing Systems (CHI'19), ACM Inc., New York, NY, 1-13. https://doi.org/10.1145/3290605.3300932

[56] Kathleen Kara Fitzpatrick, Alison Darcy, and Molly Vierhile. 2017. Delivering cognitive behavior therapy to young adults with symptoms of depression and anxiety using a fully automated conversational agent (Woebot): A randomized controlled trial. FMIR Mental Health 4, 2 (Apr. 2017), e19. DOI: https://doi.org/10. 2196/mental.7785

[57] Mauro De Gennaro, Eva G. Krumhuber, and Gale Lucas. 2020. Effectiveness of an empathic chatbot in combating adverse effects of social exclusion on mood. Front. Psychol. 10 (Jan. 2020), Article 3061, 14 pages. DOI: https://doi.org/10.3389/ fpsyg.2019.03061

[58] Naomi Aoki. 2020. An experimental study of public trust in AI chatbots in the public sector. Gov Inf Q. 37, 4 (Oct. 2020), 101490. DOI: https://doi.org/10.1016/j. giq.2020.101490

[59] Junhan Kim, Yoojung Kim, Byungjoon Kim, Sukyung Yun, Minjoon Kim, and Joongseek Lee. 2018. Can a machine tend to teenagers' emotional needs? A study with conversational agents. In Extended Abstracts of the 2018 CHI Conference on Human Factors in Computing Systems, ACM Inc., New York, NY, 1-6. https: //doi.org/10.1145/3170427.3188548

[60] Ashish Viswanath Prakash and Saini Das. 2020. Intelligent conversational agents in mental healthcare services: A thematic analysis of user perceptions. Pacific Asia Journal of the Association for Information Systems 12, 2 (June 2020), Article 1. DOI: https://doi.org/10.17705/1pais.12201

[61] Nan Hu, Paul A. Pavlou, and Jennifer Zhang. 2006. Can online reviews reveal a product's true quality? Empirical findings and analytical modeling of online word-of-mouth communication. In Proceedings of the 7th ACM conference on Electronic commerce. ACM Inc., New York, NY, 324-330. https://doi.org/10.1145/ 1134707.1134743

[62] Lise Tevik Løvseth and Olaf G Aasland. 2010. Confidentiality as a barrier to social support: A cross-sectional study of Norwegian emergency and human service workers. Int. f. Stress Manag. 17, 3 (Mar. 2010), 214-231. DOI: https: //doi.org/10.1037/a0018904

[63] Qian Yu, Tonya Nguyen, Soravis Prakkamakul, and Niloufar Salehi. 2019. "I almost fell in love with a machine": Speaking with computers affects self-disclosure. In Extended Abstracts of the 2019 CHI Conference on Human Factors in Computing Systems (CHI'19). ACM Inc., New York, NY, 1-6. https://doi.org/10.1145/3290607. 3312918

[64] Yi-Chieh Lee, Naomi Yamashita, Yun Huang, and Wai Fu. 2020. "I hear you, I feel you": Encouraging deep self-disclosure through a chatbot. In Proceedings of the 2020 CHI conference on human factors in computing systems. ACM Inc., New York, NY, 1-12. https://doi.org/10.1145/3313831.3376175

[65] Nancy L Collins and Lynn Carol Miller. 1994. Self-disclosure and liking: A metaanalytic review. Psychological Bulletin 116, 3 (Apr. 1994), 457-475. DOI: https: //doi.org/10.1037/0033-2909.116.3.457

[66] Renwen Zhang. 2017. The stress-buffering effect of self-disclosure on Facebook: An examination of stressful life events, social support, and mental health among college students. Comput. Hum. Behav. 75, 10 (May 2020), 527-537. DOI: https: //doi.org/10.1016/j.chb.2017.05.043 
[67] Pratyusha Kalluri. 2020. Don't ask if artificial intelligence is good or fair, ask how it shifts power. Nature 583, (July 2020), 7815. DOI: https://doi.org/10.1038/d41586020-02003-2

[68] Jessica Vitak and Nicole B. Ellison. 2013. 'There's a network out there you might as well tap': Exploring the benefits of and barriers to exchanging informational and support-based resources on Facebook. New Media Soc. 15, 2 (July 2012), 243-259. DOI: https://doi.org/10.1177/1461444812451566

[69] Petter Bae Brandtzaeg, Antoine Pultier, and Gro Mette Moen. 2019. Losing con trol to data-hungry apps: A mixed-methods approach to mobile app privacy. Soc. Sci. Comput. Rev. 37, 4 (May 2018) 466-488. DOI: https://doi.org/10.1177/ 0894439318777706

[70] Adam N. Joinson. 2008. Looking at, looking up or keeping up with people? Motives and use of Facebook. In Proceedings of the SIGCHI conference on Human Factors in Computing Systems (CHI'08). ACM Inc., New York, NY, 1027-1036. https://doi.org/10.1145/1357054.1357213

[71] Petter Bae Brandtzæg, Jan Heim, and Amela Karahasanović. 2011. Understanding the new digital divide-A typology of Internet users in Europe. Int. 7. Hum. Comput. Stud. 69, 3 (Mar. 2011), 123-138. DOI: https://doi.org/10.1016/j.ijhcs.2010. 11.004

[72] Bryan Marshall, Peter Cardon, Amit Poddar, and Renee Fontenot. 2013. Does sample size matter in qualitative research?: A review of qualitative interviews in
IS research. 7. Comput. Inf. Syst. 54, 1 (Dec. 2015), 11-22. DOI: https://doi.org/10. 1080/08874417.2013.11645667

[73] Glenn A. Bowen. 2008. Naturalistic inquiry and the saturation concept: A research note. Qual. Research 8, 1 (Feb. 2018), 137-152. DOI: https://doi.org/10.1177/ 1468794107085301

[74] Douglas Ezzy. 2013. Qualitative Analysis. Routledge. New York, NY.

[75] Amanda L. Forest and Joanne V. Wood. 2012. When social networking is not working: Individuals with low self-esteem recognize but do not reap the benefits of self-disclosure on Facebook. Psychol. Sci. 23, 3 (Feb. 2012), 295-302. DOI: https://doi.org/10.1177/0956797611429709

[76] Sherry Turkle. 2011. Alone Together: Why We Expect More from Technology and Less from Each Other. Basic Books, New York, NY.

[77] Curtis D. Hardin and Terri Conley. 2001. A relational approach to cognition: Shared experience and relationship affirmation in social cognition. In Hardin, C. D., Conley, T. D., \& Moskowitz, G. B. (Eds.), Cognitive SocialPsychology: The Princeton Symposium on the Legacy and Future of Social Cognition (pp. 3-17). Lawrence Erlbaum Associates Publishers, Mahwah, NJ.

[78] Marita Skjuve and Petter Bae Brandzaeg. 2019. Measuring user experience in chatbots: An approach to interpersonal communication competence. In Bodrunova, S. et al. (Eds.) Internet Science. INSCI 2018. Lecture Notes in Computer Science, Vol. 11551. Springer, Cham. https://doi.org/10.1007/978-3-030-17705-8_10 